\title{
Actividad Física y sedentarismo: Repercusiones sobre la salud y calidad de vida delas personas mayores

\author{
Physical activity and sedentary lifestyle: Impact on health and quality of life of older people
}

\author{
*Francisco Salinas Martínez; Armando Cocca; Kamal Mohamed y Jesús Viciana Ramírez \\ *Universidad de Granada (España)
}

\begin{abstract}
Resumen: Realizamos un análisis del estado actual de las personas mayores en relación con la actividad física y el sedentarismo, éste último aspecto convertido en la actualidad como una de las principales fuentes de amenaza para la salud pública de los países europeos y americanos. Damos a conocer los efectos negativos de la inactividad física sobre los parámetros cardiovasculares, diabetes, depresión y cáncer, entre otros. Mostramos los beneficios de la actividad física (herramienta clave para solucionar los problemas propios del sedentarismo) para la salud de este colectivo de la población; al tiempo que estudiamos las razones por las que las personas mayores acuden a los programas de actividad física. Finalmente, aportamos una serie de conclusiones.. Palabra clave: Actividad Física. Calidad de Vida. Personas mayores. Salud. Sedentarismo.
\end{abstract}

Abstract: We analyzed the current status of the elderly in relation to physical activity and sedentary, the latter now become a major source of threat to public health in the European and American countries. We report the negative effects of physical inactivity on cardiovascular parameters, diabetes, depression and cancer, among others. We show the benefits of physical activity (a key tool to solve the problems of the sedentary lifestyle) for the health of this group of the population while we study the reasons why older people attend physical activity programs. Finally, we provide a number of conclusions.

Key words: Physical Activity. Quality of Life. Elderly. Health. Sedentary.

\section{Introducción}

Según datos derivados de la Organización Mundial de la Salud (2002), el número de personas que superan los 60 años de edad está en incremento. En el año 2025 habrá unos 1200 millones de personas mayores de 60 años en el mundo de los cuales, 840 millones vivirán en países en desarrollo (OMS, 2002). A estos datos, hay que añadir que Europa se encuentra entre los continentes que más envejecen, pudiendo llegar en el 2050 a un 37\% de personas mayores (Unión Europea, 2002); datos similares a los que se alcanzarán en España (IMSERSO, 2004).

En este sentido, la práctica de actividad física en el colectivo de las personas mayores debe convertirse en una obligación (Salinas et al., 2007), estando ello respaldado no sólo por numerosos estudios (Martínez del Castillo et al., 2008), sino también por la administraciones (Ramos y Pinto, 2005), las cuales consideran de gran importancia la presencia de programas de actividad física dirigidos a este sector de la población.

\section{Antecedentes}

La realización de cualquier tipo de práctica deportiva está directamente relacionada con el bienestar, los estilos de vida saludables, así como la mejora de la salud y calidad de vida (Landi et al., 2007; Rebollo, 2008).

Los beneficios que aporta la realización de actividad física para el estado de salud y calidad de vida (Marcos y Galiano, 2004), siendo estos aún más evidentes en la tercera edad y los riesgos que conllevan los estilos de vida sedentarios, propios sobre todo de las personas mayores, han sido demostrados de manera notoria en numerosos estudios epidemiológicos (Guayar et al., 2004; Chodzko-Zajko, 2006; Salinas et al., 2007). En cambio, pese al incremento producido en los últimos años

\footnotetext{
Fecha recepción: 15-01-10 - Fecha envío revisores: 15-01-10 - Fecha de aceptación: 15-01-10 Correspondencia: Francisco Salinas Martínez

C/ Huerto, 82 bajo dech

14650 Bujalance (Córdoba)

E-mail: pacosalinas@ugr.es
}

en el número de personas mayores que realizan actividad física, éste continua siendo insuficiente (Gonzalo y Pasarín, 2004; García Ferrando, 2006; Martínez del Castillo et al., 2009), siendo una de sus principales causas las diferentes tipos de barreras con que se encuentra este sector de la población (Jiménez-Beatty, 2002; Martínez del Castillo et al., 2007). Por ello, hoy día el sedentarismo de nuestra sociedad es amenaza constante para la salud pública (Varo y Martínez-González, 2006; Varo, et. al., 2003), lo que está llevando a los profesionales procedentes del ámbito sanitario a adoptar medidas encauzadas a la promoción de la actividad física de la sociedad en general y las personas mayores en particular.

\section{El sedentarismo propio de las personas mayores}

La práctica de actividad física realizada de manera regular aporta toda una serie de beneficios para la salud de las personas mayores (Montoya, 2005). Por contra, los estilos de vida sedentarios, propios sobre todo de la tercera edad, están asociados a la aparición de riesgos que afectan negativamente a la calidad de vida de este colectivo (Varo et al., 2003a).

El sedentarismo de nuestra sociedad se está convirtiendo en una amenaza constante para la salud pública (Varo y Martínez-González, 2006), lo que está llevando a los profesionales procedentes del ámbito sanitario a adoptar medidas encauzadas a la promoción de la actividad física de la sociedad en general y las personas mayores en particular. En Estados Unidos, la falta de actividad física y el tabaco son las dos causas principales de muerte en este país (McGinnis y Foege, 1993). La Organización Mundial de la Salud, recoge en su «Informe sobre la Salud en el mundo 2002», que los estilos de vida sedentarios son una de las diez causas fundamentales de muerte y discapacidad en el mundo (World Health Organization, 2002). Esta misma organización, incluye entre los objetivos de salud para todos en el año 2010, el incremento del número de individuos que realizan actividad física moderada de forma regular (Organización Mundial de la Salud, 2001; World Health Organization, 2003). En el estudio «Global Burden of Disease Study» (estudio sobre la Carga Global de Enfermedad), se calcula que la inactividad física 
constituye la octava causa de mortalidad en el mundo y representa un $1 \%$ de la carga total de enfermedad (Murray y López, 2002).

Afortunadamente, en los últimos años se ha producido un incremento en el número de personas mayores que realizan actividad física, aunque éste continua siendo escaso e insuficiente (Gonzalo y Pasarín, 2004; Martínez del Castillo et al., 2009). Esta misma tendencia está presente en Europa y España, donde la población es cada vez más sedentaria debido entre otros aspectos a la mayor disponibilidad de medios de locomoción y la práctica de un tipo de ocio pasivo (Varo et al., 2003a). La sociedad actual está haciendo uso de estilos de vida sedentarios hasta alcanzar unos límites alarmantes e intolerables (Varo et al., 2003a). Asimismo, estamos asistiendo a un incremento notable en el número de personas mayores existentes en la sociedad y se estima que este aumento sea progresivo en los últimos años. Según las conclusiones derivadas de la segunda Asamblea Mundial sobre el Envejecimiento (Madrid, 2002), para el año 2050 se estima que el colectivo de personas mayores se haya triplicado. Los poderes públicos tienen que adoptar por tanto, medidas al respecto para cuidar de la salud de las personas mayores, con el objeto de que éstos puedan llevar a cabo su vida con plena autonomía (Merino, 2002).

\section{Efectos negativos de la inactividad física}

Numerosos estudios epidemiológicos han constatado que la falta de actividad física y el sedentarismo afecta negativamente a la salud de la sociedad en general (Varo et al., 2003b) y de las personas mayores en particular (Greeg et al., 2003; Varo et al., 2003b). En cambio, la práctica de ejercicio físico repercute positivamente sobre el estado de salud de las personas mayores (Talbot et al., 2002).

La diabetes es uno de los aspectos sobre los que repercute el sedentarismo (Wei et al., 2000). Un 91\% de los casos de diabetes tipo 2 pueden evitarse adoptando un estilo de vida en presencia de la actividad física de forma regular (Hu et al., 2001). Folsom et al. (2000), realizó un estudio de cohortes con una población de 34.257 mujeres de entre 55 y 69 años y obtuvo que las mujeres activas tenían un riesgo relativo de diabetes de 0.69 respecto a las sedentarias. Además, el ejercicio físico es un eslabón fundamental para que las personas diabéticas puedan alcanzar un control metabólico adecuado (Kirk et al., 2003). Sus beneficios son notorios incluyo a baja intensidad (Kirk et al., 2003).

A nivel cardiovascular la inactividad física está asociada a enfermedades tales como hipertensión arterial, accidentes cerebrovasculares y cardiopatía isquémica (Varo et al., 2003b), ésta última, principal causa de muerte en Europa y en los países desarrollados (World Health Organization, 2002). En diversas investigaciones se ha demostrado que la práctica controlada de ejercicio físico, disminuye la presión sistólica y diastólica de las personas sedentarias (Writing group of the PREMIER Collaborative Research Group, 2003), lo que se traduce en una notoria disminución del número de muertes asociadas a enfermedades cardiovasculares (Whelton et al., 2002). Estudios llevados a cabo con más de 5.000 sujetos muestran que las personas sedentarias tienen mayor riesgo de padecer hipertensión arterial (Pereira et al., 1999) y cardiopatía isquémica (Myers, 2002). La actividad fisica se convierte en uno de los protectores principales para luchar contra la cardiopatía isquémica tanto en hombres (Manson, 2002), como en mujeres (Manson, 2002). En estudio realizado con más de 9.500 ancianas estadounidenses se encontró una relación directa entre estilo de vida y mortalidad (Greeg et al., 2003). Las mujeres que iniciaron un estilo de vida activo tuvieron mucho menos riesgo de mortandad.
El ejercicio físico también posee efectos terapéuticos en el tratamiento de la depresión y la ansiedad, mejorando entre otros aspectos las funciones cognitivas de las personas (Fox, 1999). Existe por tanto una relación inversa entre la práctica de ejercicio y la presencia de síntomas asociados a la depresión y ansiedad (Strawbridge, et al., 2002).

El sedentarismo y la falta de ejercicio físico, está también ligado a la aparición y mantenimiento de la obesidad(DiPietro, 1999). En Europa, el sedentarismo es el principal responsable de la ganancia de peso en la sociedad (Aranceta et al., 2003). Posiblemente, el sedentarismo y la inactividad física sean los dos factores principales del incremento notorio en el número de personas obesas registrado en EE.UU y Europa (Varo et al., 2003a).

La presencia de actividad física en la tercera edad, disminuye la pérdida de masa ósea en general, lo que repercute en una disminución del número de caídas tan frecuentes en esta edad (Batty, 2002; Gregg et al., 2003;). Según diversos estudios, las personas mayores que realizan ejercicio físico sufren menor número de caídas, lesiones y fracturas que los ancianos sedentarios (Gregg et al., 2003; Rogers et al., 2003). Además, las personas mayores que siguen algún programa de ejercicio ingresan en menos ocasiones en los centros hospitalarios (Perkins y Clark, 2001), lo que se traduce en una mejor calidad de vida para este colectivo y en una notoria reducción de los costes sanitarios para el gobierno (Stearns et al., 2001).

Igualmente, el porcentaje de padecer cáncer puede llegar a reducirse hasta un 40-50\% (Hardman, 2001) cuando la persona practica ejercicio físico (Thune y Furberg, 2001). Aunque bien es cierto que la actividad física por sí sola no es capaz de prevenir el desarrollo de una enfermedad de este tipo (Varo et al., 2003b). Actualmente no existen suficientes evidencias científicas que justifiquen este hecho.

Conviene por tanto, analizar las actitudes y estados de cambio por los que pasan las personas hasta alcanzar un hábito de vida activo estable y permanente (Varo et al., 2003b). Todo ello con el objeto de modificar los hábitos y comportamientos de las personas mayores (Varo et al., 2003b). No podemos olvidar que el sedentarismo es un problema que afecta a la sociedad desde su infancia hasta su madurez (Greeg et al., 2003; Varo et al., 2003b). Debemos, comenzar a paliar este problema desde la base, esto es, comenzando a concienciar desde los centros educativos de la importancia que tiene la práctica de la actividad física independientemente de la etapa de la vida en que se encuentre la persona.

La actividad física como solución a los problemas del sedentarismo

El abandono de los hábitos negativos presentes en el estilo de vida de las personas mayores de nuestra sociedad (Stephens y Craig, 1990), es hoy día el principal reto contra el que se debe luchar desde la administración pública. La práctica de ejercicio físico se convierte en el medio más saludable de que disponemos para tratar de acabar con este grave problema (Solum y Handa, 2002; Talbot et al., 2002), lo cual, está ampliamente constatato (Hart et al., 2008; Liu-Ambrose y Donaldson, 2009).

Desde el punto de vista de la resistencia, está constatado que un programa de 15 semanas de ejercicio físico aeróbico mejora la resistencia cardiovascular de las personas mayores, aspecto éste imprescindible para prevenir gran parte de las enfermedades de nuestra población anciana (Hernández, 2001). Montoya (2005), realizó un programa de actividad física aeróbica sistemática con personas mayores, cinco veces 
a la semana (de lunes a viernes), durante seis meses. El grupo experimental a pesar de no llevar un control dietético disminuyó el índice de masa corporal, mejoró la resistencia cardiopulmonar e incrementó la fuerza. Todo ello es un aspecto imprescindible para lograr una mejor calidad de vida como medio de estabilización y prevención de enfermedades (Montoya, 2005). Existe mucha variabilidad a la hora de prescribir un programa de ejercicio físico cardiovascular para las personas mayores (Salinas y Viciana, 2006), aunque todos ellos repercuten positivamente en el estado de salud de los ancianos (Montoya, 2005; Pont y Roca, 2005).

Es conocido que la fuerza que es capaz de generar una persona varía con la edad, siendo menores las mejoras de fuerza en personas de edad avanzada que en sujetos de inferior edad (Martínez y Padilla, 2005). Si tenemos en cuenta el sexo, las mujeres manifiestan en mayor medida que los hombres el descenso de la fuerza (Frontera, Hughes y Evans, 1991). Además, la disminución de la masa muscular está asociada por regla general con estilos de vida sedentarios en adultos (Vandervoort y Symons, 2001). Por ello, el incremento de masa muscular y de fuerza obtenido mediante la práctica de actividad física reduce el riesgo de desarrollar enfermedades músculo esqueléticas, conduciendo auna mejora de su calidad de vida (Westhoff, Stemmerk y Boshuizen, 2000). Anivel general, el entrenamiento de fuerza en personas mayores se traduce en una serie de mejoras entre las que destacan (Martínez y Padilla, 2005): mejora del tono muscular, mejora de la masa muscular, mejora de la coordinación intramusculare intermusculary mejoras neuronales. Desde un punto de vista específico, el trabajo de fuerza en ancianos disminuye el efecto envejecimiento, mejora la independencia funcional y la calidad de vida, reduce el riesgo de patologías osteoporóticas y articulares, disminuye la cantidad de grasa corporal, mejora la capacidad de control postural y del equilibrio, reduce el riesgo de caídas y fracturas, etc (Martínez y Padilla, 2005). Según una revisión realizada por Layne y Nelson (1999), los estudios científicos realizados hasta el momento indican que el entrenamiento de la fuerza muscular está asociado positivamente con una alta densidad mineral ósea en jóvenes y ancianos.

\section{Conclusiones}

A la vista de los resultados, resaltamos la importancia de la actividad física, como elemento fundamental para que las personas mayores puedan mantener un estado óptimo de salud, así como la principal herramienta de educación y prevención de complicaciones que puedan afectar su esperanza de vida.

Nos encontramos por tanto, en una época donde la actividad física es una herramienta indispensable para reduciry paliar las preocupaciones de las personas mayores, al tiempo que regenera su vitalidad y les dota de un estado de vida con mayor calidad.

\section{Referencias bibliográficas}

Aranceta J, Pérez Rodrigo C, Serra Majem L, Ribas Barba L, Quiles Izquierdo J, Vioque J, et al. (2003). Prevalencia de la obesidad en España: resultados del estudio SEEDO 2000. Medicina Clínica (Barcelona), 120, 608-612.

Batty GD. (2002). Physical activity and coronary heart disease in older adults. A systematic review of epidemiological studies. European Journal of Public Health, 12, 171-176.

Chodzko-Zajko, W. (2006). The Usa National Strategic Plan for promoting physical activity in the mid-life and older adult population. Studies in Physical Culture \& Tourism, 13, 15-18Gonzalo, E. y Pasarín, M.A. (2004). La salud de las personas mayores. Gaceta Sanitaria, 18 (Supl 1), 69-80.

DiPietro, L. (1999). Physical activity in the prevention of obesity: current evidence and research issues. Medicine Sience and Sports Excersice, 31 (Suppl), S542-S546.

Folsom AR., Kushi LH., \& Hong CP. (2000). Physical activity and incident diabetes mellitus in postmenopausal women. American Journal of Public Health, 90, 134-138.

Fox KR. (1999). The influence of physical activity on mental wellbeing. Public Health Nutrition, 2, 411-418.

Frontera, WR.; Hughes, VA. y Evans, MJ. (1991). A croos-sectional study of upper and lower extremiy muscle strength in 45-78 year old men and women. Journal of Applied Physiology, 71, 644-650.

García Ferrando, M. (2006). Posmodernidad y Deporte: Entre la individualización y la masificación. Encuesta sobre hábitos deportivos de los españoles 2005. Madrid: CSD-CIS.

Gregg EW., Cauley JA., Stone K., Thompson TJ., Bauer DC., Cummings SR., et al. (2003). Relationship of changes in physical activity and mortality among older women. Journal of the American Medical Association, 289, 2379-2386.

Guayar, P.; Santa-Olalla, P.; Banegas, J.R.; López, E. y RodríguezArtalejo F. (2004). Actividad fisica y calidad de vida de la población adulta mayor en España. Medicina Clínica (Barcelona), 123, 606610.

Hardman AE. (2001). Physical activity and cancer risk. Proccedings of the Nutrition Society, 0, 107-113.

Hart, L.E.; Haaland, D.A.; Baribeau, D.A.; Mukovozov, I.M. \& Sabljic ,F. (2008). The relationship between exercise and osteoarthritis in the elderly. Clinical journal of sport medicine 18(6):508-21.

Hu FB., Manson JE., Stampfer MJ., Colditz G., Liu S., Solomon CG., et al. (2001). Diet, lifestyle, and the risk of type 2 diabetes mellitus in women. New England Journal of Medicine, 345, 790-807.

Imserso (2004) Las personas mayores en España. Informe2004. Madrid, 2004.

Jiménez-Beatty, J.E. (2002). La demanda de servicios de actividad fisica de las personas mayores, Tesis Doctoral, Universidad de León.

KirkA., Mutrie N., McIntyre P. \& Fisher M. (2003). Increasing physical activity in people with type 2 diabetes. Diabetes Care, 26, 11861192.

Landi, F., Onder, G., Carpenter, I., Cesari, M., Soldato, M., \& Bernabei, R. (2007). Physical activity prevented functional decline among frail community-living elderly subjects in an international observational study. Journal of Clinical Epidemiology, 60, 518524.

Layne, J.E.\& Nelson, M.E.(1999). The effects of progressive resistance training on bone density. Medicine Science Sports Exercise, 31 (1), 25-30.

Liu-Ambrose,T. \& Donaldson, MG. (2009). Exercise and cognition in older adults: is there a role for resistance training programmes. British journal of sport medicine 43(1):25-7.

Manson JE, Greenland P, LaCroix AZ, Stefanick ML, Mouton CP, Oberman A, et al. (2002). Walking compared with vigorous exercise for the prevention of cardiovascular events in women. New England Journal of Medicine, 347, 716-725.

Marcos Becerro J.F., y Galiano D. (2004). El Envejecimiento y sus Problemas. ElEjercicio comoSolución deAlgunos deEllos. Ejercicio, 
salud y longevidad. Sevilla. Consejería de Turismo y Deporte, 3334.

Martínez, M. y Padilla, J.M. (2005). Entrenamiento y desarrollo de la fuerza en personas mayores. Revista de Educación Física. Renovar la Teoría y la Práctica, 97, 23- 30.

Martínez del Castillo, J., Campos, A., Jiménez-Beatty, J. E., Del Hierro, D. et al., (2008). Encuesta nacional sobre actividad fisica y mayores en España (II). Sport Managers, 58, 48-52.

Martínez del Castillo, J., González Rivera, M. D., Jiménez-Beatty Navarro, J. E., Graupera Sanz, J. L., et al., (2009). Los hábitos de actividad física de las mujeres mayores en España. Revista Internacional de Ciencias del Deporte, 14, 81-93.

Martínez del Castillo, J., Jiménez-Beatty Navarro, J. E., Campos, A., Del Hierro, D., Martín Rodríguez, M., Gonzalez, M. D. (2007). Barreras organizativas y sociales para la práctica de actividad física en la vejez. Motricidad. European Journal of Human Movement, 19, 13-35.

McGinnis JM. y Foege WH. (1993). Actual causes of death in the United States. Journal of the American Medical Association, 270:2207-12.

Montoya, JH. (2005). Análisis de la condición física, en dos grupos de personas entre los 60 a 69 años, uno que hace actividad física aeróbica sistemática y otro que no lo hace, en el área urbana del Municipio de Popayán, Cauca. Revista Digital de Educación Física y Deportes. Disponible en: www.efdeportes.com/efd91/condic.htm.

Murray CJL. \& López AD. (2002) Assessing health needs: the Global Burden of Disease Study. En: Detels R, McEwen J, Beaglehole R, Tanaka H, editors. Oxford textbook of public health. 4thed. Oxford: Oxford University Press, 243-254.

Organización Mundial de la Salud (2002). Physical Activity and Older People. Ginebra: O.M.S.

Pereira MA, Folsom AR, McGovern PG, Carpenter M, et al., (1999). Physical activity and incident hypertension in black and white adults: the Atherosclerosis Risk in Communities Study. Preventive Medicine, 28, 304-312.

Perkins, A.J. \& Clark, D.O. (2001). Assessing the association of walking with health services use and costs among socioeconomically disadvantaged older adults. Preventive Medicine, 32, 492-501.

Pont, P. y Roca J. (2005). Efectos de un programa de actividad física sobre la memoria en personas mayores. Apunts: Educación Física y Deportes, 81, 105. Tesis Doctoral.

Ramos, P. y Pinto, J.A. (Coords.) (2005). Actividad fisica y ejercicio en los mayores. Madrid: Instituto de Salud Pública - Comunidad de Madrid.

Rebollo J. Estudio cualitativo y comparativo sobre los beneficios en salud de un programa de natación terapéutica. Fisioterapia 2008; 30 (5): 213-222

Salinas, F. y Viciana, J. (2006). Prescripción del ejercicio cardiovascular en la tercera edad. ICongreso Internacional de Avances en Ciencias de la Actividad Física y el Deporte, Sevilla.

Solum, D.T. \& Handa, R.J. (2002). Estrogen regulates the development of brain-derived neutrophic factor mRNA and protein in the rat hippocampus. Journal of Neuroscience, 22, 2650-2659.

Stearns, S.C., Bernard, S.L., Fasick, S.B., et al. (2001). The economic implications of self-care: the effect of lifestyle, functional adaptations, and medical self-care among a national sample of Medicare beneficiaries. American Journal of Public Health, 90, 1608-1612.
Stephens, T. \& Craig, C.L. (1990). The well being of Canadians. Highiligts of the 1988. Campbell's Survey. Ottawa: Canadian Fitness and Lifestyle Research Institute, 1-123.

Strawbridge WJ., Deleger S., Roberts RE. \& Kaplan GA. (2002). Physical activity reduces the risk of subsequent depression for older adults. American Journal of Epidemiology, 156, 328-334.

Talbot, L.A., Morrell, C.H., Metter, E.J. \& Fleg, J.L. (2002). Comparison ofcardiorespiratory fitness versus leisure time physical activity as predictors of coronary events in men aged $<$ or $=65$ years and $>65$ years. American Journal of Cardiology, 89, 1187-1192.

Thune, I. y Furberg, A.S. (2001) Physical activity and cancer risk: doseresponse ad cancer, all sites and site-specific. Medicine Science and Sports Exercise, 33 (Suppl.), S530-S550.

Vandervoort, A.A. \& Symons TB. (2001). Functional and metabolic consequences of sarcopenia. Cadian Journal of Applied Physiology, $26(1), 90-101$.

Varo, J.J., Martínez, J.A. \& Martínez-González, M.A. (2003a). Beneficios de la actividad fisica y riesgos del sedentarismo. Medicina Clínica (Barcelona), 121, 665-672.

Varo, J.J.; Martínez-González, M.A.; De Irala-Estévez, J.; Kearney, J; Gibney, M. \& Martínez, J.A. (2003b) Distribution and determinants of sedentary lifestyles in the European Union. International Journal of Epidemiology, 32, 138-146.

Varo, J.J. \& Martínez-González, M.A. (2006). El consejo sanitario en la promoción de la actividad física. Medicina Clínica (Barcelona), 0 , $0-0$.

Wei, M., Gibons, L.W., Kampert, J.B. et al. (2000). Low cardiorespiratory fitness and physicl inactivity as predictors of mortality in men wth type 2 diabetes. Annals of Internal Medicine, 132, 605-611.

Westhoff, MJ.; Stemmerk, L. \& Boshuizen, HC. (2000). Effects of a low-intensity strength-training program on knee-extensor stregth and functional ability of frail older people. Journal of Aging and Physical Activity, 8, 325-342.

Whelton SP., Chin A., Xin X. y He J. (2002). Effect of aerobic exercise on blood pressure: a meta-analysis of randomized, controlled trials. Annals of Internal Medicine, 136, 493-503.

World Health Organization. World Health Report (2002). Geneva: World Health Organization, 2002.

Writing group of the PREMIER Collaborative Research Group (2003). Effects of comprehensive lifestyle modification on blood pressure control. Journal of the American Medical Association, 289, 20832093.

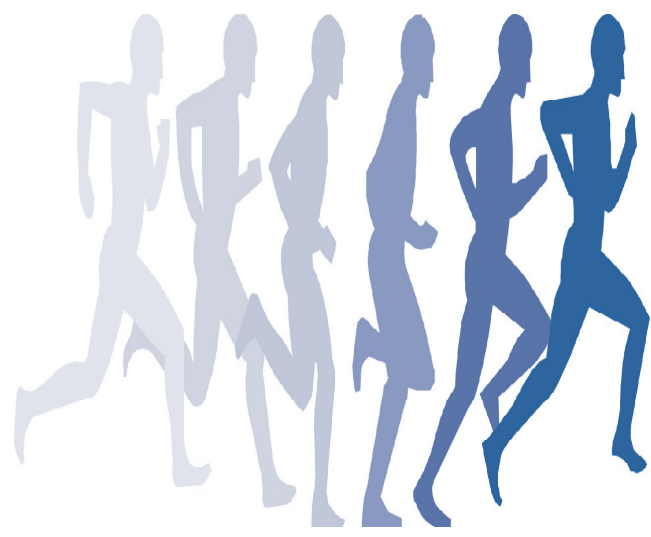

